\title{
LES PETITS PAS DE LA PAIX. NOTE SUR LE COURANT “HUMANE EDUCATION"
}

Jérôme Michalon

De Boeck Supérieur | Education et sociétés »

2018/1 n 41 | pages 121 à 127

ISSN 1373-847X

ISBN 9782807391833

Article disponible en ligne à l'adresse :

https://www.cairn.info/revue-education-et-societes-2018-1-page-121.htm

\section{Pour citer cet article :}

Jérôme Michalon, « Les petits pas de la Paix. Note sur le courant "Humane Education” », Education et sociétés 2018/1 (n41), p. 121-127.

DOI 10.3917/es.041.0121

Distribution électronique Cairn.info pour De Boeck Supérieur.

(C) De Boeck Supérieur. Tous droits réservés pour tous pays.

La reproduction ou représentation de cet article, notamment par photocopie, n'est autorisée que dans les limites des conditions générales d'utilisation du site ou, le cas échéant, des conditions générales de la licence souscrite par votre établissement. Toute autre reproduction ou représentation, en tout ou partie, sous quelque forme et de quelque manière que ce soit, est interdite sauf accord préalable et écrit de l'éditeur, en dehors des cas prévus par la législation en vigueur en France. Il est précisé que son stockage dans une base de données est également interdit. 


\title{
Les petits pas de la Paix Note sur le courant "Humane Education"
}

\author{
Jérôme MICHALON \\ Triangle (UMR 5206, CNRS, ENS de Lyon, \\ Université Lyon 2, IEP de Lyon, \\ Université Jean Monnet Saint-Étienne) \\ ENS de Lyon site Descartes, Bat D4 \\ 15 parvis René Descartes \\ 69342 Lyon cedex 07 \\ <jerome.michalon@ens-lyon.fr>
}

T e 20 février 2017, une tribune "Enseignons à l'école l'empathie pour les animaux !" est parue dans Libération, signée par seize personnalités, universitaires pour la plupart, vétérinaires, philosophes, juristes ou biologistes. Les enfants, disent-ils, ont une empathie naturelle pour les animaux, qu'ils considèrent spontanément comme des "sujets d'une vie". L'école doit donc être le lieu où cet élan est cultivé et valorisé. Ce n'est pas le cas pour les signataires qui critiquent la représentation des animaux dans les programmes scolaires (insistant sur la notion d'espèce et non d'individus animaux), le manque de réflexions philosophiques et éthiques sur la condition animale et la participation à des activités (zoos, cirques notamment) qui entérinent une séparation radicale et une hiérarchie entre humains et animaux. Rappelant que des références à l'éthique animale apparaissaient dans les manuels scolaires français de 1883 à 1923, ils citent la Belgique contemporaine comme exemple à suivre et demandent que les enseignements d'éducation morale et civique intègrent de plein droit la question animale.

La justification avancée est claire : "L'expérience que fait l'élève de la condition animale favorise ainsi chez le futur citoyen le sens des responsabilités et de la coopération, le rejet de la violence et aussi des discriminations arbitraires entre humains." Éduquer à la bientraitance des animaux aurait donc des vertus morales, favorisant l'instauration de relations interpersonnelles pacifiées. Cette association entre éducation au respect des animaux et pacification des mœurs est la marque de fabrique de ce que le monde anglo-saxon nomme "Humane Education". Ce mouvement, décrit plus loin, s’inscrit dans la continuité des premières entreprises 
de protection animale, qui ont toujours été porteuses d'un discours sur les vertus propédeutiques du respect de l'animal.

\section{Le souci moral de la protection animale : contagion et inflation de la violence}

T 'histoire de la protection animale est désormais bien connue (Agulhon 1981, Carrié 2015, Pierre 1997, Pelosse 1981, 1982). C'est au XIX ${ }^{\mathrm{e}}$ siècle, d'abord en Angleterre, puis en Allemagne, en Autriche ou encore en France, que se créent les premières sociétés de protection, qui initient tout un travail d'influence de l'opinion publique et du monde politique pour faire adopter des législations pénalisant les mauvais traitements faits aux animaux domestiques. Dire que le mouvement de protection animale était porteur d'un projet moral concernant les humains fait presque figure de truisme, mais il est utile d'en tracer les grandes lignes. Pour les membres des sociétés protectrices de l'époque, dont la composition sociologique se situe du côté de la bourgeoisie, le respect des animaux est un moyen d'éduquer le peuple et, par-delà, de civiliser la société. La peur de la violence des classes populaires est très présente dans les milieux aisés, surtout dans la France du début du XIX ${ }^{\mathrm{e}}$ siècle où l'instabilité politique renforce ce sentiment d'insécurité. La maltraitance animale est vue comme le symptôme évident de ce climat délétère : les premiers protecteurs dénoncent, par exemple, les jeux traditionnels violents, les combats d'animaux organisés par des garçons bouchers et les coups infligés par les charretiers à des chevaux éreintés de fatigue. Deux éléments motivent alors les velléités protectrices. D'une part, la crainte d'une forme de contagion de la violence : il faut éviter que ces mauvaises mœurs à l'égard des animaux puissent se diffuser socialement, gangréner des parties des milieux populaires encore indemnes. D'autre part, une inflation de la violence à contenir : l'idée est bien ancrée que la maltraitance animale est la première étape vers la violence interpersonnelle, plus particulièrement celle subie par les enfants et les épouses. L'un des fondateurs de la SPA française écrivait en 1846 : "Comment des hommes si prompts à s'irriter sans raison contre des animaux ne le seraient-ils pas à s'irriter contre leurs semblables ?" (cité par Agulhon 1981, 90). Dès lors, il s'agit d'abord de bannir la visibilité de la maltraitance animale. La loi Grammont (1850), première loi de protection animale en France, interdit ainsi les mauvais traitements infligés aux animaux dans l'espace public. Forme de compromis stratégique ? Première étape d'un combat plus vaste ? Cette priorité donnée à la publicité des mauvais traitements informe sur une conception de la protection axée au moins autant sur la répression que sur la prévention. Traïni 
(2011) souligne à juste titre la place de la prévention lors de la création en 1824 de la première association au monde la "Royal Society for The Prevention of Cruelty to Animals".

\section{La philosophie "Humane"}

Oete ette orientation préventive est au cœur du courant "Humane" qui se développe aux États-Unis dans la seconde moitié du XIX siècle, signant au passage les débuts de la protection animale sur le continent américain. La forme que prennent ces initiatives est originale : sous le nom généraliste de "ligues anticruauté", la plupart de ces organismes se donnent pour objet à la fois la protection animale et la protection de l'enfance (Pearson 2011). Dans un environnement où la protection de l'enfance n'existe pas encore, ces organismes ont utilisé les pouvoirs de police que leur mission de protection animale leur octroie pour soustraire des enfants à la maltraitance familiale. C'est donc une approche transspécifique (i.e. qui dépasse les différences entre les espèces) de la protection qui est promue, mettant en avant une vulnérabilité partagée par les enfants humains et les animaux domestiques, placés dans des situations de subordination, les condamnant à être les objets d'une violence "de toute puissance" selon l'expression souvent utilisée dans l'univers Humane. Protéger cette double vulnérabilité est conçu comme un devoir moral, allant de pair avec l'éducation des enfants à la bientraitance des animaux : la "Humane Education". Cette conception trouve ses racines dans ce que Unti et De Rosa (2003) nomment l'éthique de la bonté envers les animaux (Kindness-to-animals ethics), dont le philosophe John Locke fut le précurseur en soulignant l'importance de surveiller et de corriger le comportement des enfants à l'égard des animaux. Dans la période postrévolutionnaire, une littérature mettant en scène des enfants respectueux des animaux, mais aussi des animaux eux-mêmes vertueux, connut un grand succès au sein de la bourgeoisie américaine naissante (Grier 1999). Il s'agissait en priorité d'éduquer les garçons, destinés à être les futurs dirigeants de la jeune nation : leur apprendre le contrôle de soi, à ne pas abuser des positions de pouvoir, à être des meneurs sans être des tyrans. Avoir un animal, l'éduquer, le dresser avec bienveillance, malgré l'asymétrie flagrante de la relation, est ainsi une manière d'éprouver des compétences de dirigeants. Après la fin de la Guerre de Sécession, au moment de la création des premières organisations Humane, le projet d'une pacification globale de la société s'ajouta au souci initial d'éduquer à la vertu. Le projet Humane se veut donc le remède au constat sur lequel il repose : pour endiguer la contagion et l'inflation de la violence, il faut privilégier la contagion et l'inflation de la bienveillance. 


\section{Le mouvement Humane en actions}

E ntre 1860 et 1920, le mouvement Humane prit de l'ampleur en accompagnant le développement aux États-Unis de l'éducation publique, gratuite et obligatoire (Unti \& De Rosa 2003). Adhérant au discours méritocratique et républicain de l'école publique, les associations Humane ont œuvré pour faire en sorte que des enseignements d'instruction morale et civique axés sur la bientraitance des animaux fassent partie des programmes scolaires. Elles publièrent énormément de livres et de manuels destinés aux enseignants, et les diffusèrent largement dans les écoles. Elles organisèrent également des concours de rédaction ou d'affiches réalisées par les écoliers, sur le thème de la paix, sous l'angle interspécifique, entre les espèces (la Fondation Waltham notamment fut très active dans ce domaine). Ou encore des actions plus concrètes comme le recueil et l'adoption collective d'animaux errants, au sein des classes, ou l'implication dans les "Bands of Mercy”, ces regroupements d'enfants et d'adolescents, agissant localement pour prévenir la maltraitance animale. Mais surtout, ces associations et leurs sections locales impulsèrent l'adoption de lois rendant obligatoire l'enseignement Humane. Ainsi, en 1920, une vingtaine d'États américains avaient adopté de telles lois. Les associations (telle l'American Humane Association) veillèrent auprès des éditeurs de manuels scolaires à ce que les éléments "Humane" apparaissent bien dans les ouvrages. En France, c'est également au sein des enseignements d'instruction civique et morale que le respect de l'animal est enseigné à partir de la fin du XIX siècle (Fédi 2008), sans qu'on connaisse précisément les raisons de cette inclusion ni de son abandon au milieu des années 1920. Aux États-Unis, la Première Guerre mondiale a reconfiguré le mouvement Humane : craignant d'être accusées d'antimilitarisme, du fait de leurs positions pacifistes, les actions Humane se donnent une coloration patriotique. Face à la guerre, le combat pour l'obligation d'éduquer à la bientraitance animale paraît dérisoire et le mouvement s'essouffle. De nombreuses associations décidèrent de se consacrer en priorité à une activité de refuge et de soins pour les animaux errants ou abandonnés. La population d'animaux en détresse ne cessant de crôitre, les actions de prévention passèrent au second plan. Fragilisé en interne, le mouvement Humane n'arrive plus à s'assurer du suivi des initiatives qu'il a portées, tant à l'échelon régional que fédéral. Le développement de la motorisation des moyens de transport rend, en outre, la maltraitance animale (incarnée par l'image du cocher ou du charretier frappant ses chevaux) moins visible, et plus abstraite pour les écoliers. L'entre-deux-guerres affaiblit les mouvements de protection animale dans leur ensemble et le mouvement Humane en particulier. À la fin de la Seconde Guerre mondiale, la présence du respect des animaux dans les programmes scolaires américains n'est quasiment plus qu'un lointain souvenir. D'une 
part, la concurrence d'autres groupes d'intérêts qui investissent l'école : le monde scientifique, l'industrie ou l'agriculture cherchent à orienter les programmes, avec une force de frappe plus conséquente que celle du mouvement Humane. D'autre part, la question du rapport aux animaux, incarnée jusqu'ici dans les enseignements d'observation de la nature et d'éducation Humane, se voit réduite à l'enseignement d'une biologie purement scientifique, excluant des notions jugées trop romantiques comme la bienveillance. Le mouvement Humane s'oriente alors vers d'autres modes d'action.

\section{“The Link" : la conviction objectivée}

D epuis les années 1970, les associations Humane ont investi le monde de la recherche, en finançant et publicisant des études visant à prouver l'existence du lien entre maltraitance animale et violences interpersonnelles. Elles promeuvent aussi les pratiques de soin et d'assistance animalières pour les personnes handicapées et/ou en souffrance (Michalon 2014).

Cet investissement a donné lieu à une abondante littérature -située entre la psychologie, la criminologie, le droit et la sociologie: Arbour, Signal et al. 2009, Ascione 1997, Ascione \& Arkow 1999, Flynn 1999, Linzey 2009-qui est identifiée à travers un mot clé : "The Link". Ce terme, déposé en copyright par l'American Humane Association (<www.americanhumane.org/>), est utilisé par de nombreux organismes Humane pour désigner autant les recherches documentant le lien entre violences interpersonnelles et celles faites aux animaux, que le mot d'ordre derrière lequel chercheurs et militants doivent se regrouper. En 2008, la National Link Coalition a été créée aux États-Unis (<http://nationallinkcoalition.org/>) par des militants Humane, des chercheurs, des psychologues, des travailleurs sociaux, des vétérinaires, avec pour objectif d'élaborer une stratégie de diffusion massive des recherches et de ses conséquences normatives et pratiques auprès de différents acteurs institutionnels et de terrain. Le but de la stratégie autour de The Link est de former des professionnels du travail social, mais aussi les policiers, à repérer lors de leurs interventions la maltraitance animale comme un indicateur de violence domestique, subie par les conjointes et les enfants. Les vétérinaires sont aussi sensibilisés à la question et renseignés sur la procédure de signalement de faits inquiétants concernant les animaux, auprès des autorités compétentes en matière de protection de l'enfance notamment. Aux Pays-Bas, vétérinaires, travailleurs sociaux et policiers ont ainsi été formés et une police des animaux, dédiée à l'identification, la prévention et la sanction de la maltraitance animale, a été créée (Enders-Slegers \& Janssen 2009). 


\section{Conclusion}

Comme son nom l'indique, le mouvement Humane accorde une importance cruciale à l'idée d'humanité. À l'instar de toute entreprise qualifiée de philanthropique, il s'agit d'encourager tout acte qui témoigne de l'humanité de la personne qui l'effectue. L'expression française "agir avec humanité" traduit bien cette performance de l'anthropos dont il est question ici. La particularité du mouvement Humane tient à l'inclusion d'êtres qui n'appartiennent pas à l'espèce humaine. Faire entrer les animaux dans l'humanité comme communauté morale et construire des relations interspécifiques pacifiées constituent son enjeu central. Dès lors, l'éducation, plus particulièrement, l'éducation publique devient le ressort essentiel de cette bataille. Le bilan des efforts du mouvement Humane est délicat à établir tant ses objectifs sont ambitieux, ses moyens d'action relativement modestes et ses modalités d'intervention très fines. Qu'en est-il aujourd'hui de l'accroissement du souci de l'animal ? D'un côté, il semble que la cause animale ait grandement gagné en légitimité et en audience depuis le début du XXI ${ }^{e}$ siècle. La bienveillance vis-à-vis des animaux est devenue un leitmotiv très consensuel : dans les débats actuels, qu'ils engagent des militants, des chasseurs, des éleveurs ou des environnementalistes, la question n'est plus de savoir s'il faut ou non respecter les animaux, mais de statuer sur la meilleure façon de le faire. De ce point de vue, il y a quelque chose d'inédit dans la situation que nous vivons aujourd'hui. C'est sans doute ce qui a encouragé les signataires de la tribune convoquée en ouverture de cette note. D'un autre côté, cette même tribune, en se référant à la place faite aux animaux dans les programmes scolaires de la III République, semble vouloir rappeler l'existence d'une époque où l'esprit du temps était tout aussi propice au respect des animaux. Les déboires du mouvement Humane invitent pourtant l'analyste à relativiser les succès des mobilisations proanimaux et à souligner à la fois la contingence de ces victoires et leur dépendance vis-à-vis des institutions auprès desquelles il est question de manœuvrer. En quoi l'institution scolaire serait-elle aujourd'hui plus qu'hier disposée à intégrer durablement le respect de l'animal dans ses missions ? Il y a là pour les spécialistes de la question matière à enquête.

\section{Références bibliographiques}

AGULHON M. 1981 "Le sang des bêtes. Le problème de la protection des animaux en France au XIX" siècle", Romantisme-31, 81-109

ARBOUR R., SIGNAL T. \& TAYLOR N. 2009 "Teaching Kindness: The Promise of Humane Éducation” Society $\mathcal{E}$ Animals-17(2), 136-148

ARKOW P. 1996 "The relationships between animal abuse and other forms of family violence", Family Violence Ė Sexual Assault Bulletin-12(1-2), 29-34 
ASCIONE F. R. 1997 "Humane education research: Evaluating efforts to encourage children's kindness and caring toward animals", Genetic Social and General Psychology Monographs-123(1), 59-77

ASCIONE F. \& ARKOW P. eds. 1999 Child Abuse, Domestic Violence and Animal Abuse: Linking the Circles of Compassion for Prevention and Intervention, West Lafayette IN, Purdue University Press

CARRIÉ F. 2015 Parler et agir au nom des bêtes : production, diffusion et réception de la nébuleuse idéologique "animaliste" (France et Grande-Bretagne, 1760-2010), Thèse de doctorat en sciences politiques. Paris X Nanterre

ENDERS-SLEGERS M.-J. \& JANSSEN M. 2009 Cirkel van geweld: verbanden tussen dierenmishandeling en huiselijk geweld, Utrecht, Universiteit Utrecht

ENSEIGNONS À L'ÉCOLE L'EMPATHIE POUR LES ANIMAUX! 2017, Libération, 20 février, en ligne, <www.liberation.fr/debats/2017/02/20/enseignons-a-l-ecole-l-empathie-pour-les-animaux_1549784>

FÉDI L. 2008 "Pitié pour les animaux : une leçon de morale laïque et ses antécédents philosophiques", Romantisme-142, 25-40

FLYNN C. P. 1999 "Exploring the link between corporal punishment and children's cruelty to animals", Journal of Marriage and the Family 61 (4), 971-981

GRIER K. 1999 "Childhood socialization and companion animals: United States, 1820-1920", Society and Animals 7(2), 95-120

LINZEY A. ed. 2009 The Link Between Animal Abuse and Human Violence, Eastbourne, East Sussex, UK, Sussex Academic Press

MICHALON J. 2014 Panser avec les animaux. Sociologie du soin par le contact animalier, Paris, Presses des Mines ParisTech

PEARSON S. J. 2011 The Rights of the Defenseless. Protecting Animals and Children in Gilded Age America, Chicago, The University of Chicago Press

PELOSSE V. 19811982 "Imaginaire social et protection de l'animal. Des amis des bêtes de l'an X au législateur de 1850", $1^{\mathrm{re}}$ partie, L'Homme 21(4), 5-33; 2 $2^{\mathrm{e}}$ partie, L'Homme 22(1), 33-51

PIERRE E. 1997 "La zoophilie dans ses rapports à la philanthropie, en France, au XIX" siècle", Cahiers d'histoire-3-4, 655-675

TRAIINI C. 2011 La cause animale (1820-1980). Essai de sociologie historique, Paris, PUF

UNTI B. \& DEROSA B. 2003 "Humane education: Past, present, and future. The State of the Animals II", Salem D.J. \& Rowan A.N., Washington D.C., Humane Society Press, 27-50 